\title{
Le premier DEA de « Langue anglaise des spécialités scientifiques et techniques », contraintes et libertés
}

Monique Mémet

\section{(2) OpenEdition}

\section{Journals}

Édition électronique

URL : http://journals.openedition.org/asp/4294

DOI : $10.4000 /$ asp.4294

ISSN : 2108-6354

Éditeur

Groupe d'étude et de recherche en anglais de spécialité

\section{Édition imprimée}

Date de publication : 1 octobre 1993

Pagination : 229-257

ISSN : 1246-8185

\section{Référence électronique}

Monique Mémet, "Le premier DEA de "Langue anglaise des spécialités scientifiques et techniques », contraintes et libertés », ASp [En ligne], 2 | 1993, mis en ligne le 02 mars 2014, consulté le 01 mai 2019. URL : http://journals.openedition.org/asp/4294; DOI : 10.4000/asp.4294

Ce document a été généré automatiquement le 1 mai 2019.

Tous droits réservés 


\title{
Le premier DEA de « Langue anglaise des spécialités scientifiques et techniques ", contraintes et libertés
}

\author{
Monique Mémet
}

1 L'enseignant de langue de spécialité joue un rôle d'interface au sein des établissements d'enseignement; les interactions entre les scientifiques et le linguiste sont en effet constantes. C'est un rôle de même nature que j'ai accepté de jouer en analysant, par le biais du thème des contraintes et des libertés, le premier Diplôme d'Étude Approfondie (DEA) de «Langue anglaise des spécialités scientifiques et techniques » : interface entre l'équipe des enseignants et celle des participants au DEA.

2 Le but de l'analyse dont il est rendu compte est assimilable à celui d'une caisse de résonance : il s'agit de faire connaître l'avis des utilisateurs, les participants au premier DEA, et de favoriser la communication. Ce travail permet d'esquisser un bilan de la première promotion. Les termes retenus pour le thème du Colloque sont porteurs de multiples connotations. Les libertés ou les contraintes sont des facteurs difficiles à isoler et, a fortiori, à étudier. J'ai cependant utilisé les réponses à un questionnaire comme prétexte à une analyse statistique. Le plan de la communication suit celui des articles de recherche, à savoir introduction, méthodes et outils, résultats et discussion.

\section{Introduction}

3 En 1991-92, une première promotion a participé au DEA de «Langue anglaise des spécialités scientifiques et techniques ». Ce DEA avait reçu l'habilitation du Ministère en juillet 1991, après de nombreuses étapes dont le Professeur Michel Perrin a rendu compte lors de précédents colloques du GERAS ${ }^{1}$. J'ai demandé à tous les participants de répondre à un questionnaire autour du thème des "Contraintes et des libertés ", thème du $14^{\mathrm{e}}$ colloque. Les réponses aux questions, qui portaient sur l'espace, le temps, les aspects financiers et psychologiques, ont été regroupées à l'aide d'un tableur. Les réponses sont 
présentées sous forme de graphiques et de tableaux, et sont brièvement commentées. Le questionnaire comportant des questions ouvertes, une analyse qualitative est proposée. L'objectif principal est de dresser un bilan de l'année d'étude vue par les utilisateurs sous l'angle des contraintes et des libertés.

\section{Méthodes et outils}

\section{Questionnaire}

4 L'outil retenu a été celui d'un questionnaire envoyé individuellement à chaque participant avec une lettre circulaire (27 participants). Ce questionnaire (cf. annexe), accompagné de la lettre, a été expédié au mois d'octobre 1992 et a été suivi d'une lettre de rappel au mois de novembre. La date d'envoi correspond à la fin du cycle du DEA, la session finale s'étant déroulée en septembre 1992.

\section{Logiciel}

5 Les réponses ont été regroupées à l'aide d'un tableur (Microsoft ${ }^{\oplus}$ Excel sur système Apple $)^{\circledast}$. Pour ceux ou celles qui ne seraient pas familiers avec le concept d'un tableur, précisons qu'il s'agit d'un logiciel qui se présente sous la forme de feuilles divisées en colonnes et en lignes; chaque croisement de ligne et de colonne constitue une cellule, dans laquelle les données sont introduites. Le logiciel mémorise les relations entre les données, et les recalcule automatiquement chaque fois qu'une donnée est modifiée. Le logiciel Excel ${ }^{\circledR}$ comporte, entre autres, des feuilles de calculs et des feuilles graphiques. Signalons enfin que les systèmes actuels permettent l'utilisation d'un tableur sans connaissances étendues en informatique.

6 Les résultats sont présentés sous forme de graphiques (camemberts ou histogrammes) réalisés avec ce logiciel.

\section{Résultats}

\section{Nombre de réponses}

7 Sur 27 envois correspondant aux 27 participants effectifs, 23 participants ont répondu, soit un taux de réponse de $85,19 \%$. Ont répondu ceux qui avaient terminé le parcours, ainsi qu'un certain nombre ayant abandonné le cycle en cours d'année. Certains candidats ayant abandonné ont rempli une partie du questionnaire : un candidat, par exemple, a répondu par l'envoi d'une lettre et n'a pas rempli le questionnaire. C'est la raison pour laquelle le nombre de questionnaires utilisables est de 22. Soit, au total, un taux de réponses au questionnaire de $81,48 \%$ (voir fig. 1). 
Figure 1. Taux de réponses au questionnaire



\section{Profil des participants}

8 Le profil des participants est dressé à partir des éléments suivants : sexe, âge, lieu de résidence, fonction principale et lieu d'exercice, nombre d'années d'enseignement, titres et diplômes. Par " participants », on entendra désormais les "répondants »: c'est-à-dire que tous les résultats portent sur les 22 questionnaires utilisables.

\subsection{Sexe}

9 Sur les 22 participants, 14 hommes et 8 femmes, soit un pourcentage respectif de 63,64\% et de $36,36 \%$ (voir fig. 2).

Figure 2. Répartition selon le sexe

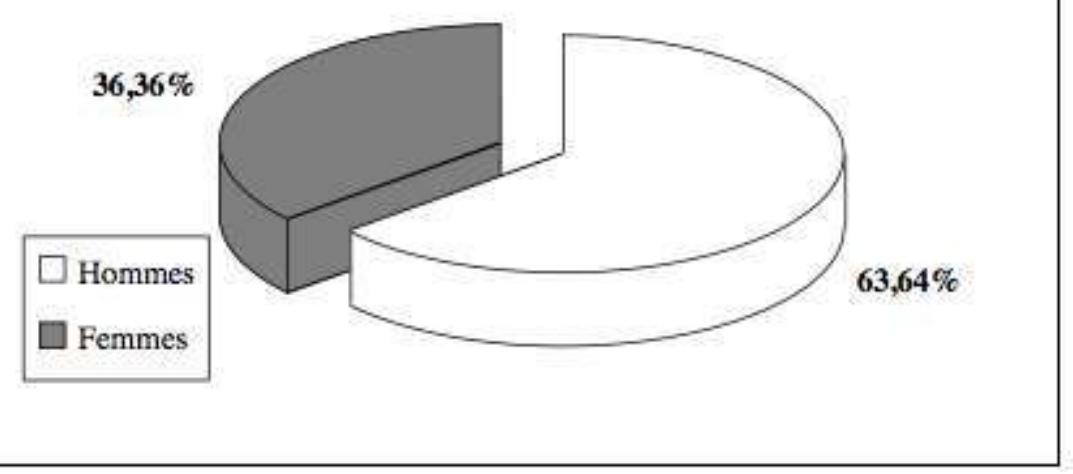

2.2. Âge

10 La majorité des participants se situe entre 41 et 45 ans (voir figure 3). L'âge moyen des participants est de 43 ans. Les âges extrêmes sont 34 et 53 ans. 
Figure 3. Âge des participants

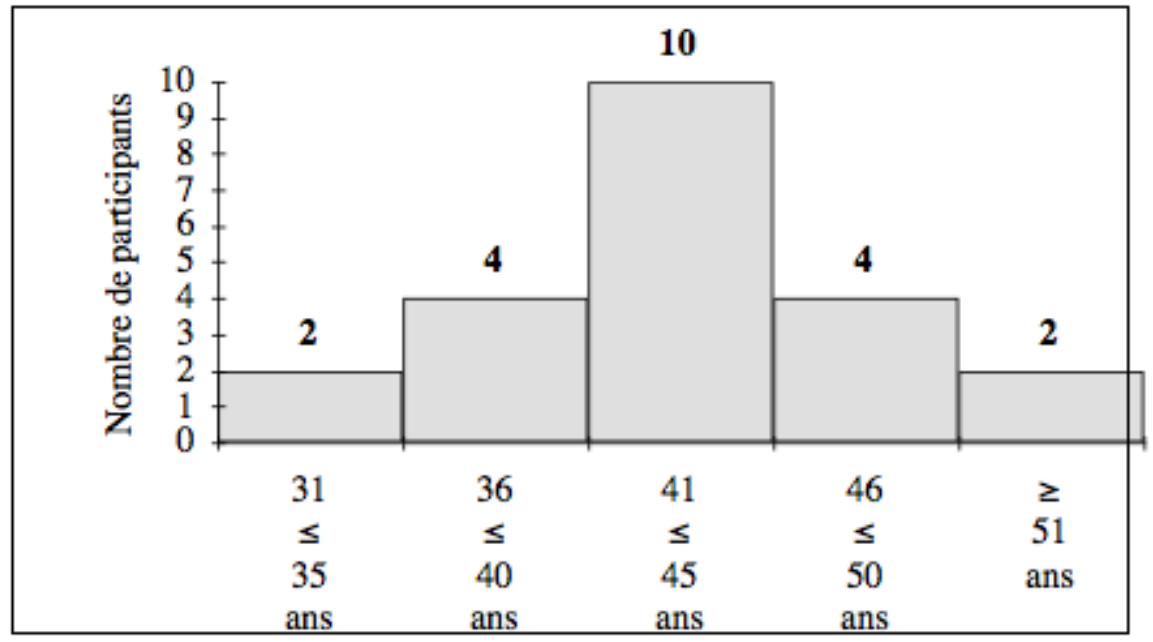

\subsection{Lieu de résidence}

11 Sur les 22 participants, 10 régions sont représentées: Aquitaine, Basse-Normandie, Bourgogne, Bretagne, Centre, Haute-Normandie, Languedoc-Roussillon, Midi-Pyrénées, Paris-Île-de-France, et Poitou-Charente. La région de Midi-Pyrénées a la plus grande représentation (6 participants) ; deux autres régions sont aussi représentées de façon plus importante : Poitou-Charente, et la région de Paris-Île-de-France (voir fig. 4).

Figure 4. Lieu de résidence des participants par région

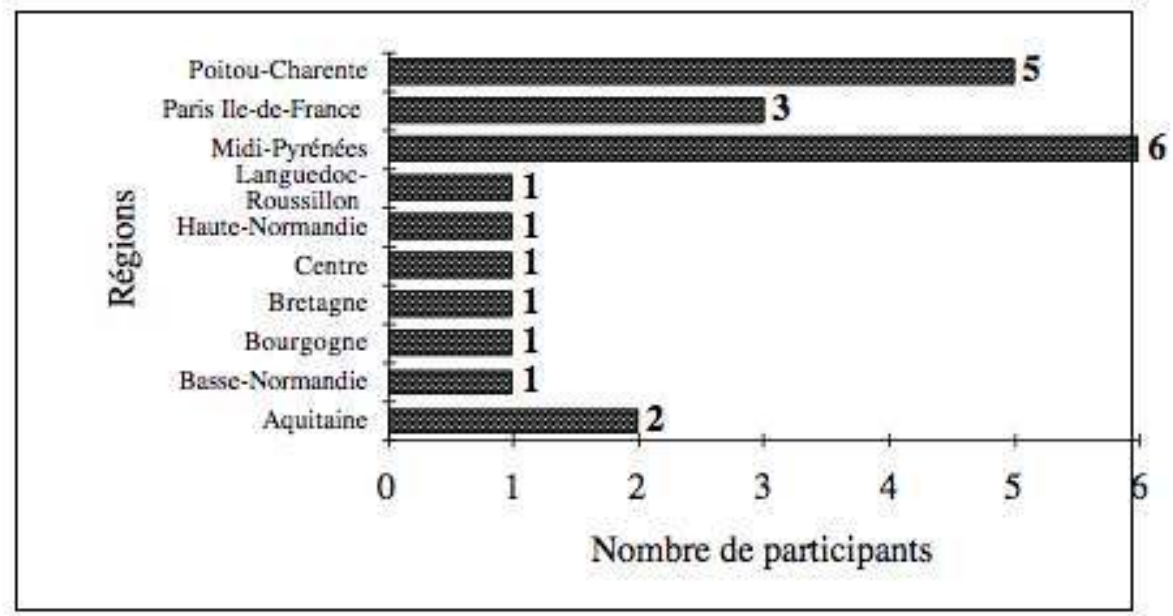

\subsection{Fonction principale et lieu d'exercice}

Tous les participants au DEA 1991-92 étaient des enseignants. La question posée portait sur la «fonction principale »; certains participants, cependant, ont répondu à la question en indiquant plusieurs types d'établissement. Il n'a pas été opéré de sélection parmi les réponses: toutes les réponses ont été retenues, c'est-à-dire 35 réponses pour 22 participants.

Les résultats font apparaître une forte proportion d'enseignants en Instituts Universitaires de Technologie (IUT), et dans différentes filières universitaires telles que LEA, UFR de Sciences - les enseignants ayant coché cette rubrique n'ont pas toujours 
précisé le secteur dans lequel ils travaillaient. Les autres types d'établissement sont les lycées, les services de Formation continue, les Grandes Écoles et les classes préparatoires. Dans la catégorie "autres", on trouve : des écoles privées (ESC, ITIP) et un IUFM (voir fig. 5). Aucun pourcentage de répartition des divers lieux d'enseignement n'est donné, car compte tenu du nombre de réponses prises en compte, le total des réponses dépasserait $100 \%$.

Figure 5. Lieu d'exercice des participants : type d'établissement

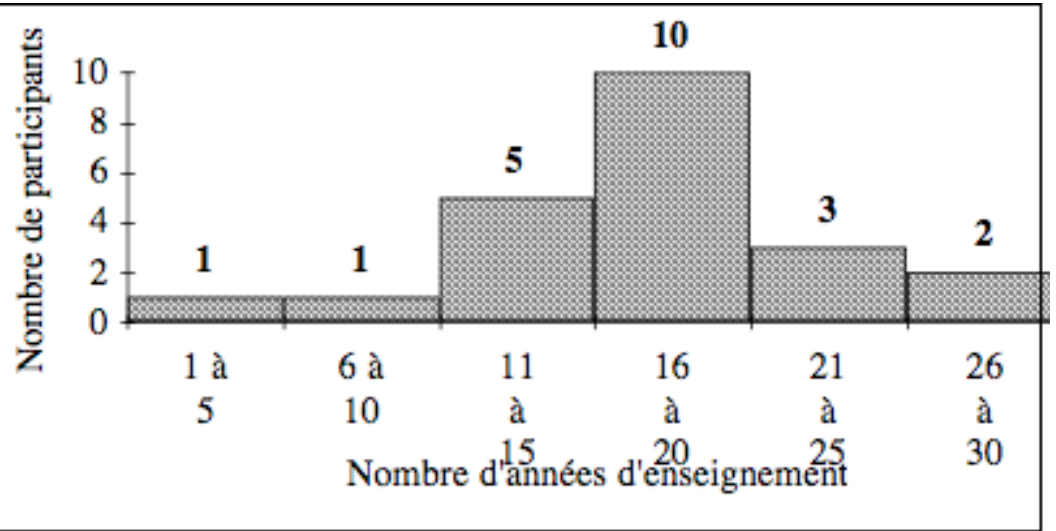

\subsection{Nombre d'années d'enseignement}

Presque la moitié des enseignants a entre 16 et 20 ans de carrière. L'ancienneté moyenne est de 18 ans avec un écart type ${ }^{3}$ de 5,6 ans. Les nombres extrêmes sont 5 et 28 ans (voir fig. 6).

Figure 6. Nombre d'années d'enseignement des participants

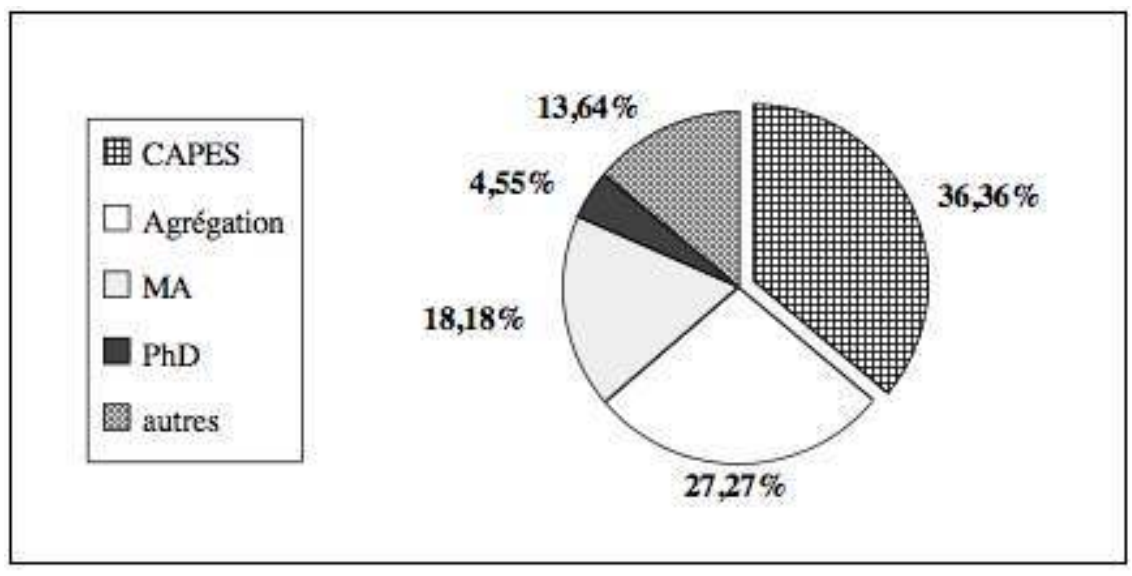

\subsection{Titres et diplômes}

La question posée portait sur les titres et diplômes. Ont été pris en compte dans les résultats le diplôme ou le titre le plus élevé. Par exemple, si le participant cochait les rubriques «CAPES » et " Agrégation », l'agrégation était retenue. Dans la rubrique divers (13,64 \%), les diplômes suivants ont été cités : B.A., licence d'anglais, maitrise, licence de biologie, DEA de littérature. La catégorie $P h D$, qui ne figurait pas en tant que telle dans le questionnaire, a été établie à partir des réponses fournies dans la rubrique « autres ». Il apparaît que la proportion la plus élevée est celle des titulaires du CAPES, 36,36\%, suivie par les titulaires de l'agrégation, 27,27 \% (voir fig. 7). 
Figure 7. Titres et diplômes des participants

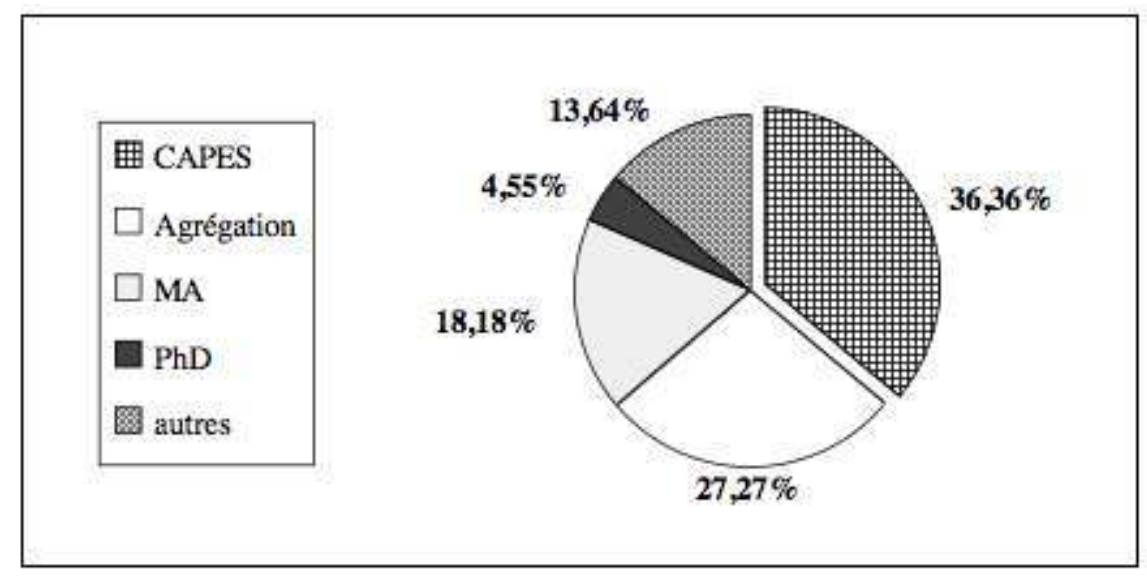

\section{Contraintes, libertés}

Les questions posées portaient sur des notions de « contraintes » et de « libertés » liées à l'espace, au temps, aux aspects financiers et psychologiques.

7 Afin de préciser le sens des termes «contrainte » et « liberté », les participants ont été invités à choisir parmi une liste des synonymes celui ou ceux qui reflétaient le mieux leur impression de contraintes, d'une part, et de libertés, d'autre part, relativement au DEA.

Les participants avaient à choisir parmi les unités lexicales suivantes ${ }^{4}$ :

Contraintes = autorité, coaction, coercition, compression, empêchement, entrave, force, gêne, obstacle, pression, violence, asservissement, assujettissement, captivité, chaîne, esclavage, joug, oppression, servitude, sujétion, tutelle

Libertés = autonomie, disponibilité, franchise, indépendance, choix, latitude, libre arbitre, licence, impunité, permission, pouvoir

En ce qui concerne les synonymes de contraintes, les participants ont retenu 12 unités sur 21 proposées. Le nombre de citations n'est pas proportionnel au nombre de participants ; il était demandé à ces derniers de choisir un maximum de 4 équivalents pour les contraintes et de 2 pour les libertés. Nous avons retenu tous les choix, quel qu'ait été le nombre de synonymes donnés. Au total, 60 citations pour les contraintes pour les 20 réponses, soit en moyenne 3 synonymes cités, et 50 citations concernant les libertés pour 21 réponses, soit en moyenne 2,3 synonymes cités.

20 Les unités citées le plus fréquemment pour les contraintes sont les unités suivantes : obstacle, gêne et empêchement, et pression. Pour les raisons mentionnées plus haut (le total des réponses dépasserait $100 \%$ ), aucun pourcentage n'est indiqué.

Les autres termes mentionnés sont par ordre de grandeur : autorité, compression, tutelle, coaction. Les termes asservissement, violence, entrave et coercition sont mentionnés chacun une fois (voir fig. 8.1). 
Figure 8.1. Synonymes de contraintes

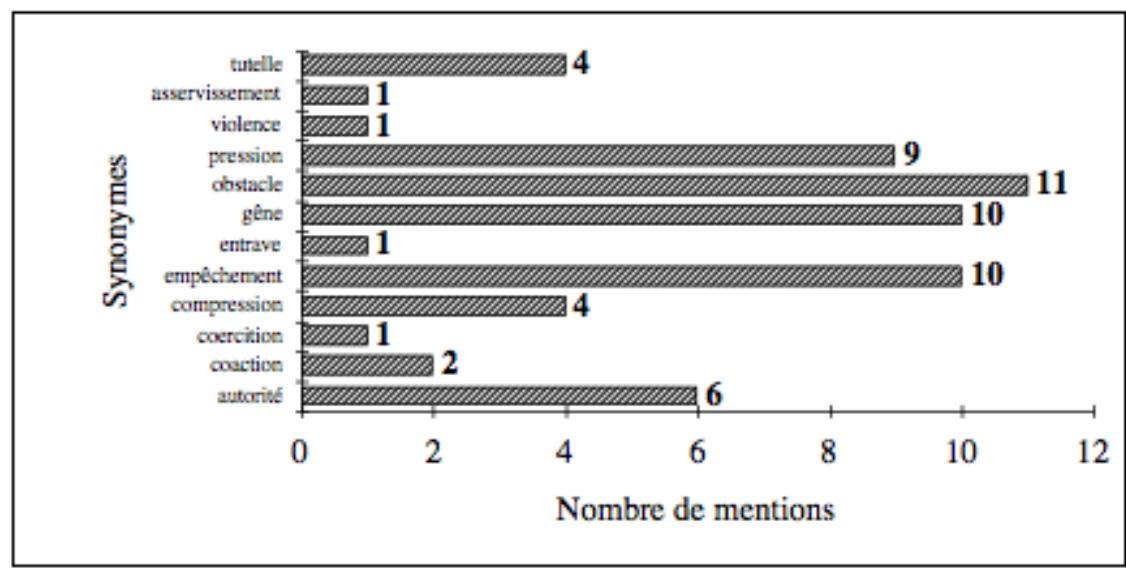

En ce qui concerne les onze termes associés au concept de liberté, 8 ont été cités. Les unités les plus fréquemment citées sont les suivantes : autonomie (15 mentions), choix (11 mentions), indépendance (8 mentions). Viennent ensuite franchise, disponibilité, pouvoir, latitude et libre arbitre (voir fig. 8.2).

Les unités suivantes n'ont pas été citées : licence, impunité, permission.

Figure 8.2. Synonymes de libertés

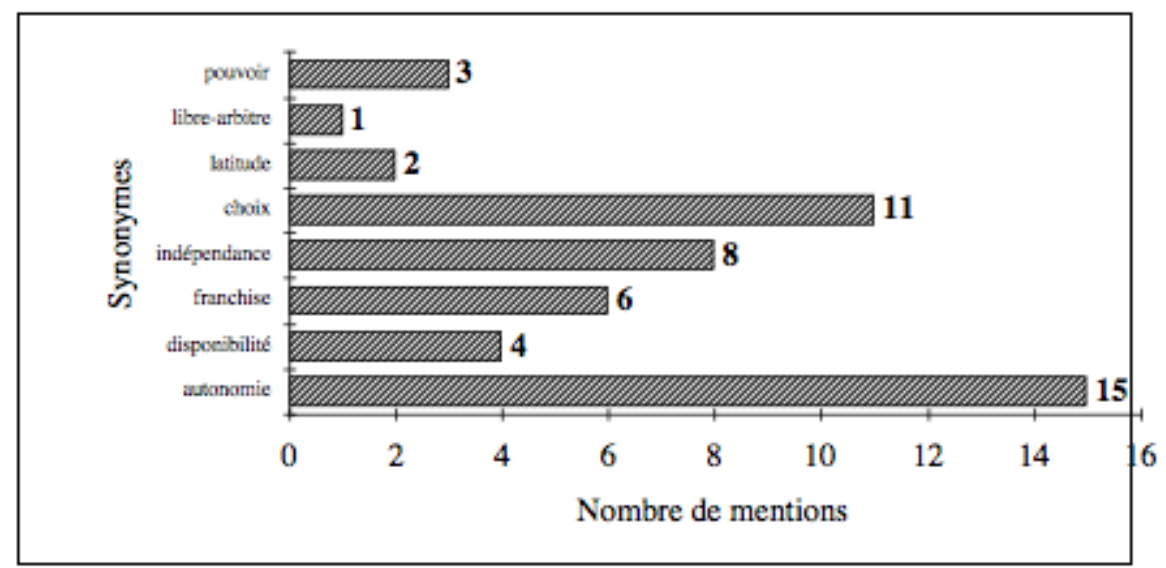

24 Les domaines concernant les contraintes et les libertés avaient été délimités dans le questionnaire. Il s'agissait de définir le degré de contrainte - ou de liberté - dans les domaines de l'espace, du temps, des aspects financiers et psychologiques, en attribuant une intensité à la contrainte ou à la liberté. Les degrés d'intensité étaient définis ainsi :

1. Pas de contrainte 1 . Liberté totale

2. Contrainte légère 2 . Liberté légèrement réduite

3. Contrainte moyenne 3. Liberté moyennement réduite

4. Contrainte relativement forte 4 . Liberté grandement réduite

5. Très forte contrainte 5 . Absence de liberté.

\subsection{Espace}

Les questions portant sur l'espace faisaient référence à la distance entre le domicile et les divers lieux de rencontre durant la période d'étude : la ville universitaire du directeur de 
recherche, le colloque et le congrès auxquels il était fortement conseillé d'assister et, si possible, d'y faire une communication. Une dernière question avait trait au jugement porté par les participants sur le degré de liberté qu'apportait la possibilité de suivre l'enseignement du DEA à distance. Les réponses aux questions du paragraphe $1 \mathrm{du}$ questionnaire «estimez-vous avoir subi des contraintes particulières?» sont données sous forme de graphes (voir fig. 9 à 9.2).

Figure 9. Contraintes espace

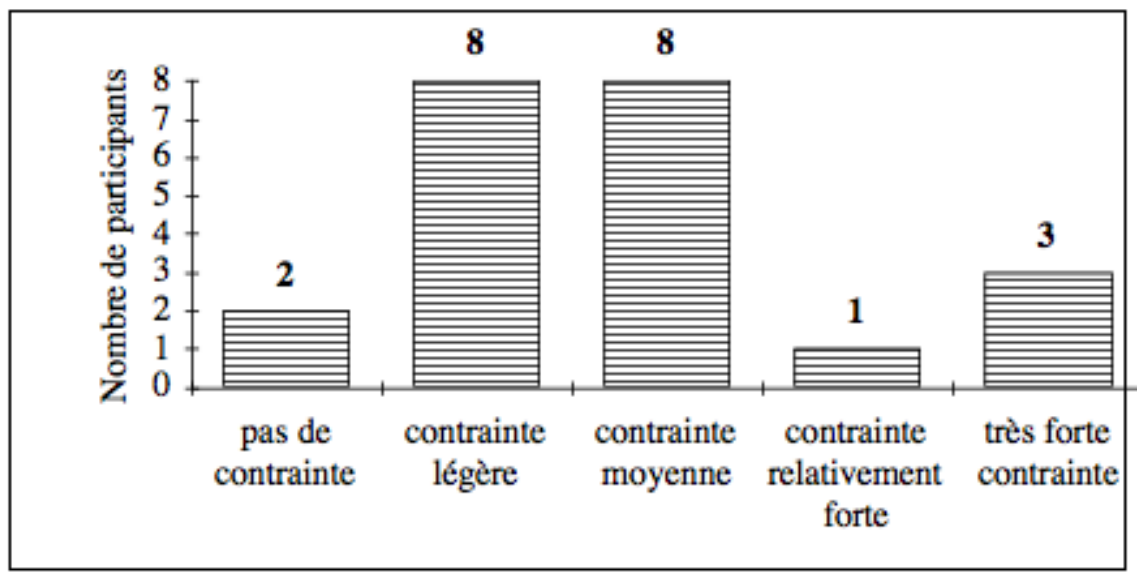

Les participants ont estimé avoir subi des contraintes nulles, légères ou moyennes dans 18 des cas. Il était demandé de préciser ces contraintes par rapport aux déplacements à effectuer. Les questions portaient sur la difficulté ou l'impossibilité de se rendre aux sessions de travail avec le directeur de recherche, au colloque du GERAS à Montpellier du 6 au 8 février 1992, et au congrès de la SAES, à Strasbourg du 15 au 17 mai 1992.

Les résultats font apparaître que la vaste majorité des participants a subi des contraintes nulles, légères ou moyennes (15 sur 17 réponses) en ce qui concerne les déplacements pour rencontrer le directeur de recherche. Pour se rendre au colloque du GERAS, la courbe se déplace vers la droite, c'est-à-dire que les contraintes relativement fortes et très fortes sont de 9 sur 18 réponses. Les résultats concernant le dernier déplacement sont encore plus nets : 15 participants sur 17 ayant répondu à la question indiquent des contraintes relativement fortes et très fortes (voir fig. 9.1). 
Figure 9.1. Contraintes espace : sessions de travail avec le directeur de recherche, colloque du GERAS, congrès de la SAES

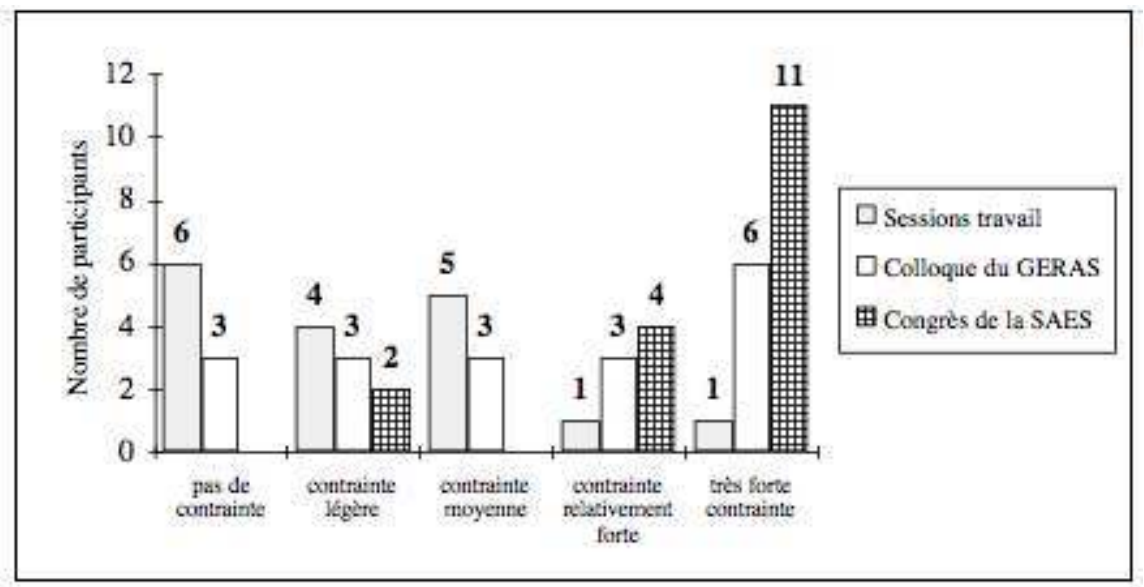

Les participants qui ont répondu que la distance n'était pas une très forte contrainte ont insisté sur la nécessité d'une très grande organisation personnelle. Des participants ont signalé l'impossibilité de se rendre au congrès de la SAES à cause de la date du congrès.

La question concernant l'espace a été posée par rapport au degré de liberté que permettait l'enseignement à distance. La question était formulée ainsi : «Estimez-vous que travailler à distance est une liberté ? ». Les réponses (voir fig. 9.2) sont le pendant de la question concernant les contraintes (fig. 9). Les participants ont estimé dans leur grande majorité (17 sur 22 réponses) qu'ils avaient bénéficié d'une liberté totale ou légèrement réduite.

Figure 9.2. Espace : liberté ou contrainte



Des participants ont noté la difficulté de disposer de certains documents immédiatement. L'espace a impliqué un isolement pour certains, se traduisant par l'absence de rencontre en cours d'année avec leurs autres collègues. Néanmoins, cet enseignement à distance leur a permis de suivre le cycle du DEA. 


\subsection{Temps}

31 La question portant sur les contraintes de temps était formulée ainsi « Estimez-vous avoir subi des contraintes particulières en matière de temps?» (paragraphe $2 \mathrm{du}$ questionnaire). Les réponses sont données à la figure 10 .

Figure 10. Contraintes temps

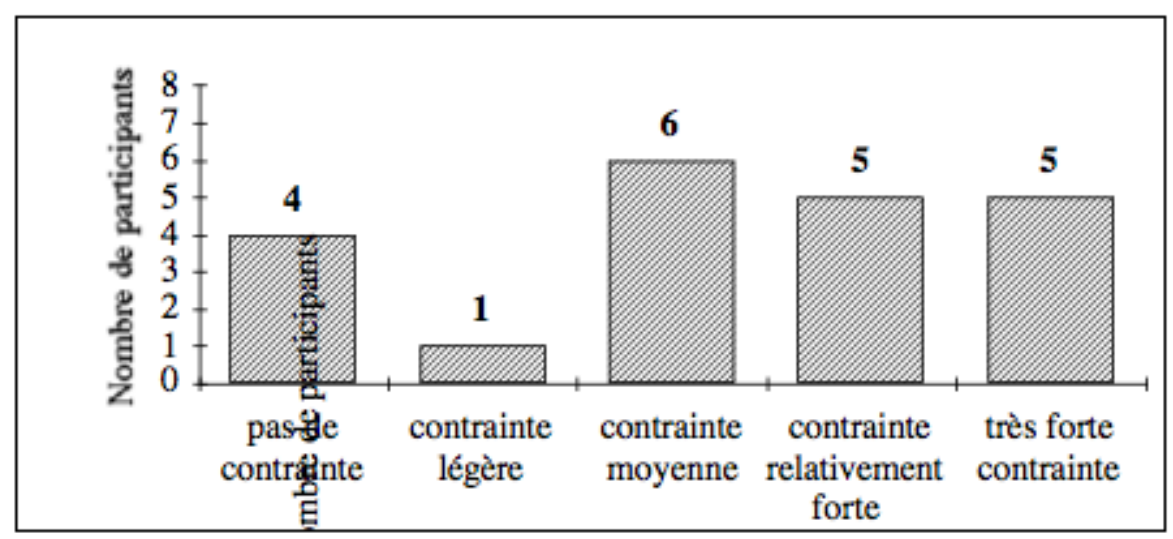

Des participants (11, soit $52 \%$ ) ont mentionné la « difficulté de mener de front les tâches d'enseignement et les remises des travaux de recherche » qui correspondent souvent à des semaines d'examens ou de partiels. Certains participants ont dû prendre des jours de congé pour se rendre aux congrès. La majorité du travail de recherche a dû être effectué en fin d'année universitaire et pour certains, après les examens ou concours de leurs étudiants, essentiellement pendant les mois de juillet et août. Aux tâches professionnelles, qu'elles soient d'enseignement ou administratives, s'ajoutent parfois des tâches familiales. D'où une difficulté pour trouver le "temps» pour le DEA. Certains participants signalent une nécessité de réduire l'activité professionnelle, et l'impossibilité de lire des ouvrages autres que ceux liés au DEA.

La nature des contraintes a été formulée par certains participants ainsi : suppression du temps «libre" pendant les congés scolaires et les week-ends. Le DEA implique l'impossibilité d'activités autres que celles de la recherche, donc un surcroît de «stress »; mais temps signifie aussi « rappel d'échéances » et, dans ce cas, les contraintes sont du ressort des contraintes psychologiques.

\subsection{Aspect financier}

34 La question portant sur l'aspect financier (paragraphe 3 du questionnaire) était formulée de façon identique à celle concernant les contraintes de temps. Tous les participants ont répondu à la question (voir fig. 11). 




Des précisions sont apportées par certains participants. Les frais de déplacement ne sont pas pris en charge par une institution (12 participants, soit $54 \%$, en font état). Sont mentionnés les frais de restauration et d'hébergement, les achats de livres, de documentation non disponibles en bibliothèques, les photocopies, le renouvellement de matériel informatique. Deux participants estiment le montant total des frais minimum (hébergement, restauration, transport, achat de livres, etc.) à environ 7000 - 8000 francs.

\subsection{Aspect psychologique}

Dix-neuf participants ont répondu à la question (paragraphe $4 \mathrm{du}$ questionnaire) concernant les contraintes psychologiques et formulée comme les questions précédentes : «Estimez-vous avoir subi des contraintes particulières d'ordre psychologique? (voir fig. 12).

Figure 12. Contraintes d'ordre psychologique

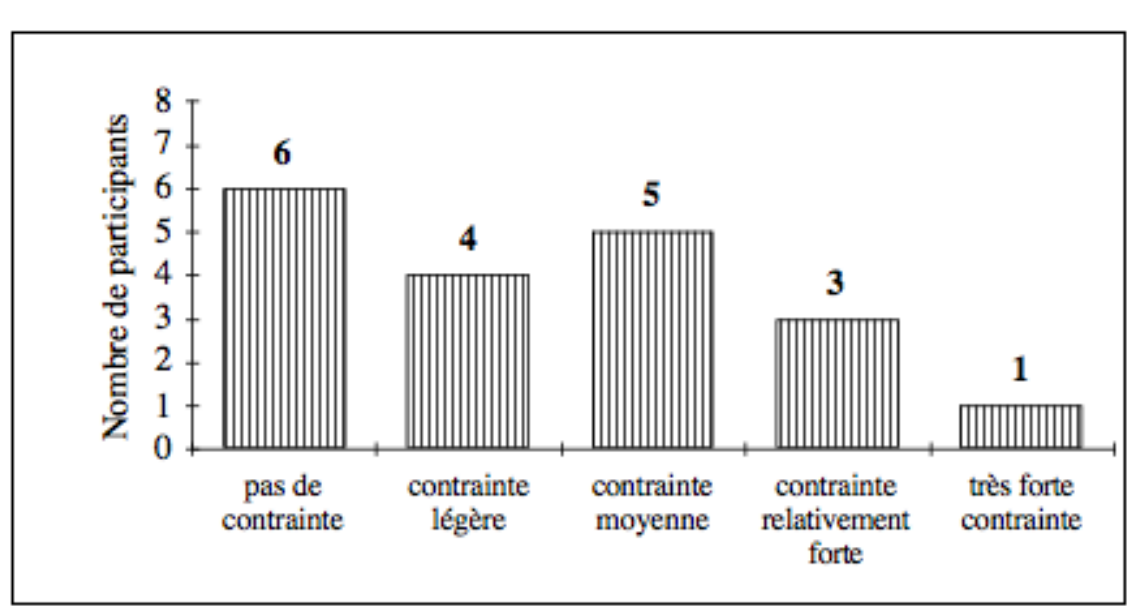

37 Pour préciser la nature ou le type des contraintes, trois questions étaient posées : le degré de contrainte concernant les relations avec le groupe de recherche, celles avec le directeur de recherche, et des contraintes personnelles. Les réponses sont représentées figures 13 à 13.2 . 
Figure 13. Contraintes - Domaine psychologique : les relations avec le groupe de recherche, avec le directeur de recherche et les contraintes personnelles

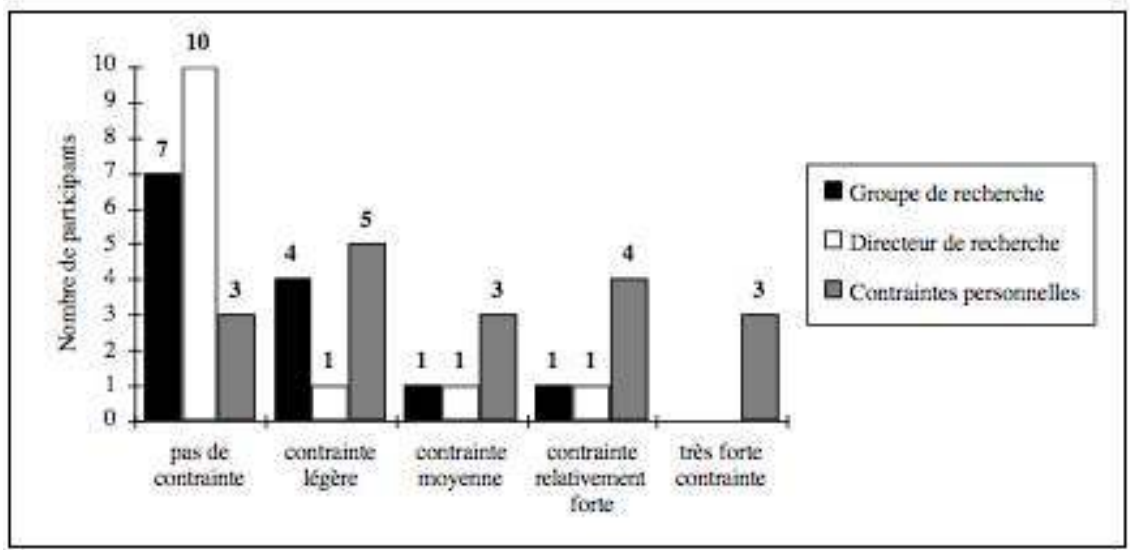

Les « contraintes personnelles » étaient précisées ensuite. Les participants répondaient par oui ou par non à la question suivante: les «contraintes concernaient-elles l'élaboration des travaux demandés", chacun de ces travaux étant ensuite défini: "élaboration et définition du sujet de recherche, rédaction de la note de recherche, rédaction des notes de lecture, soutenance de la note de recherche $»^{5}$ (voir fig. 13.1).

Figure 13.1. Contraintes personnelles

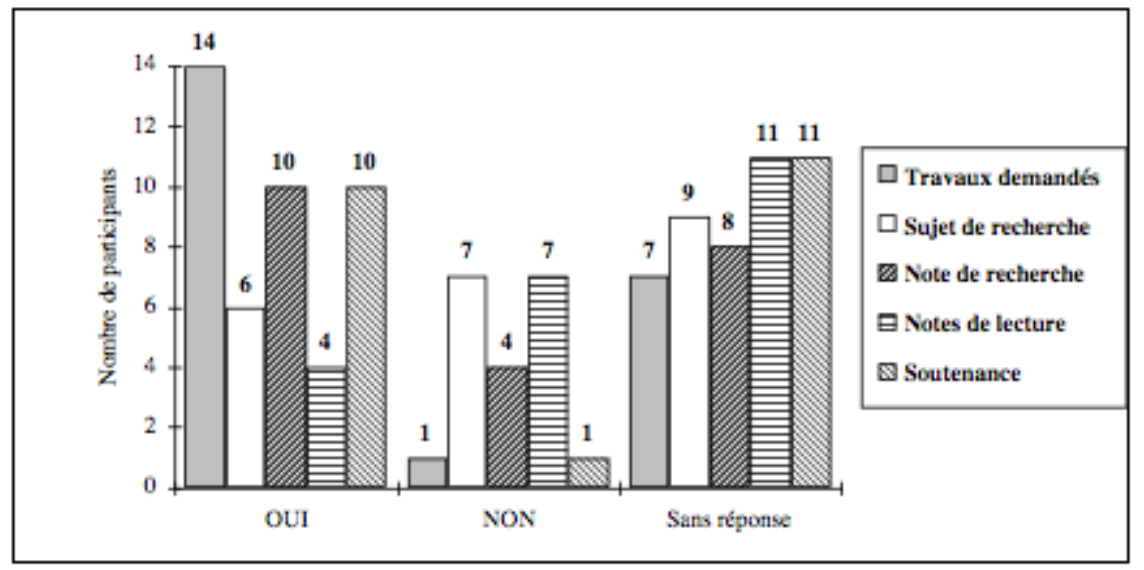

Certains participants ont indiqué plus de détails en ce qui concerne la nature des contraintes d'ordre psychologique. Ils ont mentionné le «stress» de l'examen, la difficulté de mener de front les travaux de recherche et les travaux quotidiens, la difficulté de s'organiser " pour ne pas se noyer dans la documentation ». Un participant a indiqué la contrainte liée à la langue, c'est-à-dire la nécessité de rédiger la note de recherche en français et non en anglais, langue maternelle du participant ${ }^{6}$. La soutenance a été mentionnée par un participant comme un exercice requérant un gros effort sur soimême.

Deux autres questions, destinées à cerner les impressions de contrainte ou de liberté des participants concernaient d'une part, la possibilité de choisir un sujet de recherche lié à son propre enseignement et, d'autre part, la possibilité de choisir le sujet et le directeur de recherche après la session de travail. Les questions étaient binaires et formulées ainsi : «Estimez-vous que les aspects suivants sont des contraintes ou des libertés : utiliser son 
travail sur le terrain dans le cadre de la recherche? Choisir sujet et directeur de recherche à la fin d'une session d'orientation générale et de méthodologie?». Les réponses sont indiquées à la figure 13.2.

Figure 13.2. Contrainte ou liberté ? La possibilité de choisir un sujet de recherche lié à l'enseignement, le choix du sujet et du directeur de recherche après la session de travail

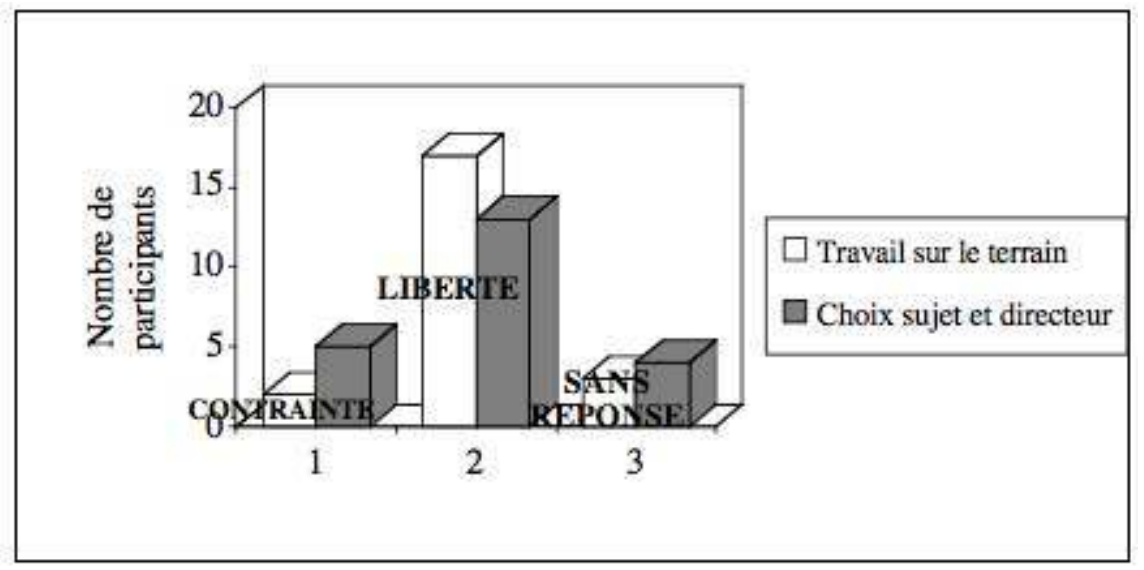

41 Il apparaît que l'utilisation de son travail sur le terrain dans le cadre de la recherche est une liberté pour 17 participants, et une contrainte pour 2. Le choix du sujet et du directeur de recherche à la fin d'une session d'orientation générale et de méthodologie est une liberté pour 13 participants, et une contrainte pour 5.

Enfin, une question concernant le degré de contrainte subi avait été posée. Il s'agissait de répondre à la question suivante: "Estimez-vous que vous n'avez pas eu assez de contraintes? » (voir fig. 14).

Figure 14. Pas assez de contraintes?

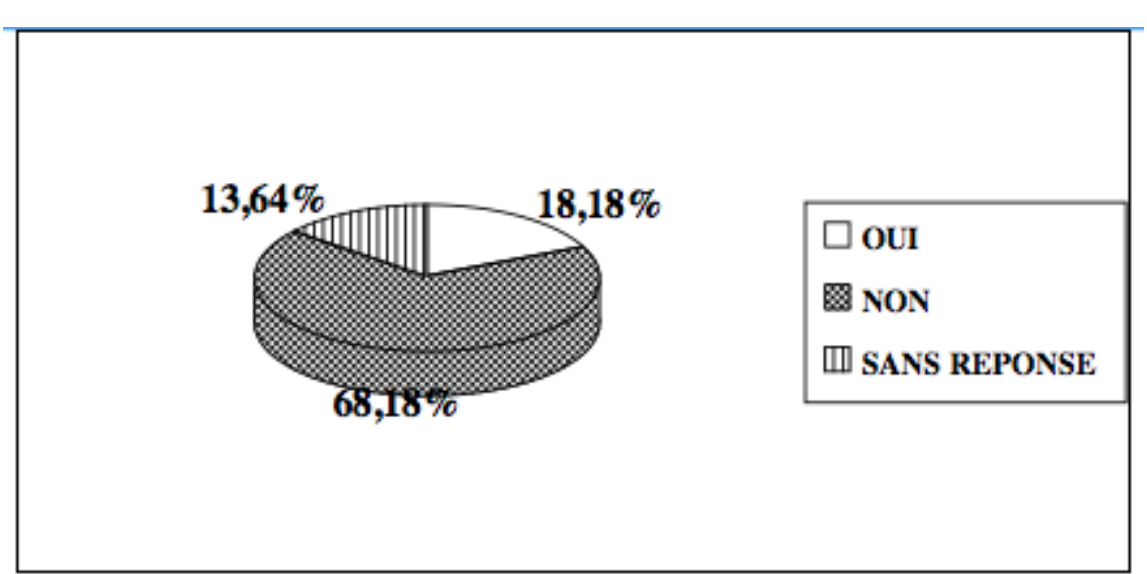

43 La question, résolument ambiguë, a suscité des réflexions diverses qui délimitent le champ des contraintes et celui des libertés. Un participant note :

Une trop grande liberté devient une contrainte dans la mesure où l'on n'est jamais certain de ce que l'on attend de nous.

Un participant indique que la forme du DEA lui a donné plus de disponibilité. Un autre fait le parallèle entre «contraintes » et «suivi » concernant en particulier la rédaction des travaux. Il eût aimé recevoir des encouragements de la part du directeur de recherche en cours de route. Les consignes sont aussi mises en rapport avec le terme de contraintes : 
l'absence de consignes concernant la rédaction de la note de recherche et le nombre non défini de comptes rendus de lecture sont assimilés à un manque ressenti de contraintes. $\mathrm{Si}$ le fait de donner des dates de remise des travaux est une contrainte dans ce cas, note un participant, c'est un élément stimulant pour un auteur.

Si l'on met en parallèle les résultats concernant les quatre types de contraintes, à savoir des domaines de l'espace, du temps, les aspects financiers et psychologiques, il apparaît que les contraintes liées à l'espace sont les moins contraignantes au même titre que les contraintes financières ou psychologiques (sur 22 réponses, 9 ou 10 " contraintes nulles » ou « légères »). Les courbes se répartissent cependant de façon différente.

Il serait aussi possible d'analyser tous ces résultats en faisant des tris croisés. Par manque de temps, et surtout à cause de la taille réduite de l'échantillon, nous ne donnerons qu'un exemple. Les réponses des hommes concernant les contraintes liées à l'espace et celles des femmes (voir figure 15).

Figure 15. Contraintes espace : réponses hommes/femmes. Exemple de tri croisé.

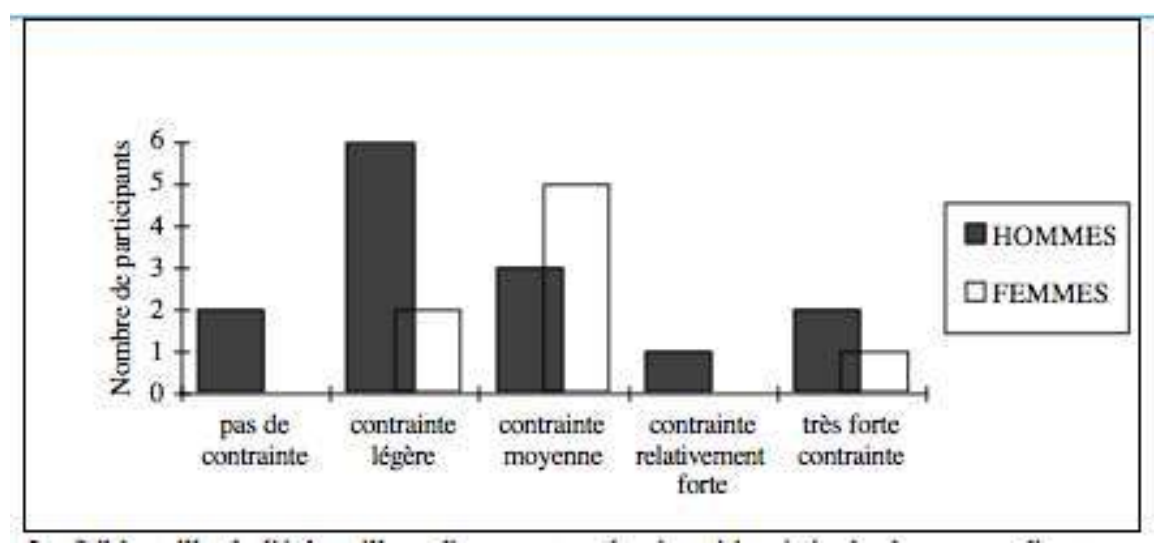

47 La faible taille de l'échantillon, d'une part, et la répartition inégale des sexes, d'autre part, ne permet pas de donner des pourcentages. Cependant, les courbes indiquent des contraintes légèrement plus importantes pour les femmes.

\section{Discussion}

\section{Nombre de réponses aux questions}

Les éléments connus avant l'envoi du questionnaire, en particulier les données concernant le sexe et le lieu de résidence, n'ont pas été pris en compte pour permettre d'avoir un véritable échantillon tout au long de l'étude.

Quant au nombre total de réponses à chaque question, il varie de 13 à 22. Cette variation ainsi que la taille de l'échantillon sont les deux raisons principales pour lesquelles les réponses ne sont pas données en pourcentages. D'autre part, si le participant répond à une question initiale d'une certaine façon en indiquant, par exemple, un nombre limité ou une absence de contraintes, il ne répond pas ensuite aux questions qui découlent de la question initiale. 


\section{Formulation des questions}

50 Il est bien connu que la façon de poser les questions influence les réponses. Les termes de contraintes et de libertés retenus pour ce colloque couvrent de vastes plages sémantiques. L'origine étymologique (Dauzat et al. 1971) éclaire les deux termes retenus pour ce colloque: contraintes du latin constringere (serrer), et libertés du latin libertas (franchises), de liber (opposé de l'esclave).

51 L'utilisation de synonymes pour préciser la charge sémantique des unités lexicales était un premier pas pour permettre d'utiliser des normes identiques. Les choix des participants en ce qui concerne les synonymes éclairent quelque peu leur perception du cycle : autonomie, choix, obstacle, gêne, empêchement et pression.

52 Le fait de faire porter le questionnaire sur le thème des libertés et des contraintes limitait au départ la liberté des réponses. Un participant le formule ainsi, d'ailleurs non sans humour:

Je trouve ce questionnaire très contraignant par sa seule insistance sur les aspects contrainte/liberté.

Certaines des questions portant sur l'espace auraient pu figurer sous la rubrique «temps ", les colloques et congrès ne se déroulant pas toujours durant des périodes de congé scolaire ou universitaire. Un participant l'a très clairement formulé ainsi :

La distance n'est un obstacle que dans la mesure où elle allonge d'autant la durée de

toute rencontre et rend difficiles les autorisations d'absence par l'administration. 
Les points mentionnés sont regroupés sous quelques rubriques par thèmes et par fréquence décroissante de citations.

\section{Remarques et suggestions formulées par les participants} ainsi que les travaux demandés. Les remarques soulignent des manques ou des inadaptations aux situations. Un terme utilisé par un des participants résume une impression générale: il faudrait «baliser le terrain ». Les candidats suggèrent que l'information (les instructions) concernant les travaux soit diffusée de manière identique entre tous les candidats. Des demandes spécifiques concernent les travaux à réaliser par les candidats au diplôme.

\section{L'examen final}

61 Il est demandé de préciser «exigences ", "règles » et "modalités de l'examen ». Des remarques sont faites concernant la forme de l'épreuve. La forme de l'examen final est «traditionnelle et [...] stressante», alors que le DEA «s'intéressait aux pédagogies nouvelles». Certains candidats posent la question de l'évaluation: "les objectifs devraient être plus explicités. Que permet l'épreuve formelle d'évaluer? Des connaissances (dans quels domaines ?), l'aptitude à la synthèse (n'est-elle pas redondante avec les autres types de travaux : notes de recherche et de lecture ?) ».

\section{La note de recherche}

Les questions posées portent sur la nature de l'exercice lui-même. Est-ce « un compte rendu de réflexions ou un produit fini ?». Certains demandent plus de «guides». Il est suggéré de limiter le volume de la production, à savoir 50 pages maximum.

Plusieurs candidats évoquent les changements intervenus lors de la session finale. Ils s'interrogent sur sa nature et son contenu; ils suggèrent de revoir le choix de certains intervenants.

Certains proposent des adaptations concernant l'organisation du cycle :

- Adjonction de cours de méthodologie en anglais de spécialité;

- Distribution d'une liste de lecture, une bibliographie, bien avant le début du cycle ;

- Regroupement des étudiants par centre d'intérêt lors de la session d'ouverture ;

- Allongement de la période pour choisir son sujet après la session d'ouverture ;

- Préparation du DEA en deux ans.

Les difficultés rencontrées par les participants provenaient pour certains d'entre eux de la difficulté de se procurer les ouvrages didactiques, d'un manque de liaison entre tuteurs et étudiants, de la non-prise en compte du calendrier de ces derniers.

Il semble que parmi ceux ou celles qui ont indiqué avoir arrêté le cycle, la contrainte essentielle ait été l'impossibilité de se rendre aux sessions; c'est-à-dire des obstacles d'espace et de temps plutôt que des obstacles psychologiques. Des raisons personnelles ont été invoquées: "contraintes extérieures», «charges d'enseignement trop importantes ", "responsabilités familiales » et des problèmes de santé. Cependant, la taille de l'échantillon ne permet pas de formuler de conclusions définitives à ce sujet. 


\section{Conclusion}

Malgré un questionnaire contraignant, il apparaît que les participants au DEA 1991-92 ont répondu librement aux questions posées et, dans leur grande majorité (voire la totalité), ils ont trouvé l'expérience enrichissante. Même si certaines critiques sont formulées sur l'organisation, il ressort une impression d'ensemble de satisfaction. Sur les 22 réponses, deux indiquent une impression nettement défavorable. Un participant insiste sur des rapports difficiles avec le directeur de recherche et conclut par : «J'estime que j'aurais pu faire un travail plus adapté et mieux préparer la soutenance, avec un peu de direction ».

Pour tous les autres participants, même si des suggestions ou critiques sont apportées, le résultat final est très positif. Le DEA permet de « repasser de l'autre côté de la barrière ", de "se mettre à la place des étudiants ». C'est une façon de "se remettre en question ", "un défi intellectuel», «le meilleur 'stage' jamais effectué », «une très bonne ambiance ». C'est une « excellente stimulation professionnelle et intellectuelle ».

Ce DEA est en effet un outil de formation, mentionné dans la presse spécialisée comme le début d'une préparation à l'Europe. Ann Johns et Tony Dudley-Evans le citent dans leur article intitulé "English for Specific Purposes: International in scope, specific in purposes" (1991: 302).

Pour conclure, il est important de considérer et d'évaluer cette formation dans le contexte général de l'étude des langues, et d'envisager nos travaux comme Claude Hagège qui désire « connaître les sociétés humaines en étudiant les langues comme un chemin de contraintes et un champ de libertés $»^{7}$.

L'auteur remercie vivement les participants à ce premier DEA qui, par leurs réponses au questionnaire, ont permis la réalisation de cette étude.

\section{BIBLIOGRAPHIE}

Butler, C. 1985. Statistics in Linguistics. Oxford : Basil Blackwell.

Chazaud, H. B. du. 1989. Dictionnaire des synonymes, Collection Les Usuels du Robert, nouvelle éd. Paris : Dictionnaires le Robert.

Dauzat, A. et al. 1971. Nouveau dictionnaire étymologique et historique. Paris : Larousse.

Johns, A. M. et T. Dudley-Evans. 1991. « English for Specific Purposes: International in scope, specific in purposes ». TESOL Quarterly 25/2, 297-314.

\section{ANNEXES}

\section{Annexe 1 : Compte-rendu des discussions}


Les questions de l'auditoire ont porté sur la nature des travaux demandés aux étudiants en DEA, sur la proportion des Francophones et des Anglophones dans le groupe, ainsi que sur des formules statistiques, et en particulier l'écart type. Les réponses à ces questions ont été insérées dans la communication sous forme de notes.

\section{Annexe 2 : Calcul de l'écart type}

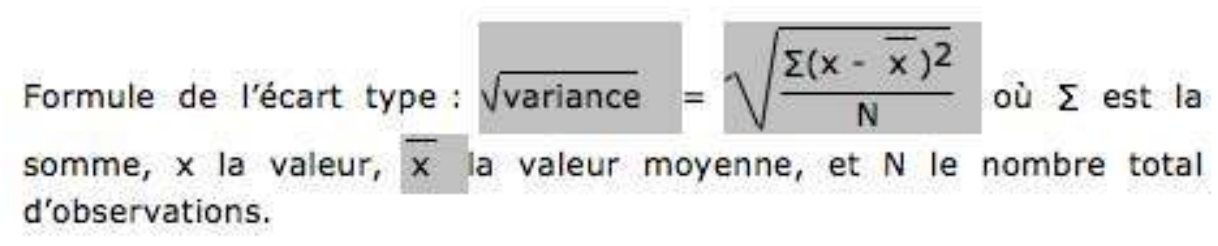

Annexe 3 : Questionnaire (voir fichier)

\section{NOTES}

1. Voir l'exposé des motifs figurant dans la demande d'habilitation, reproduit dans la Lettre circulaire $n^{\circ} 1$ aux candidats potentiels du DEA d'anglais de spécialité du 4/7/1991, ainsi que dans ESP France Newsletter 4 déc. 1992, p. 4-6.

2. Microsoft Excel est une marque déposée de la société Microsoft Corp. Apple est une marque déposée de la société Apple Computer, Inc.

3. Voir annexe 2 pour le calcul de l'écart type.

4. Tirées de Chazaud (1989).

5. Les consignes figurant dans La lettre circulaire $n^{\circ} 2$ aux candidats du DEA, du 24/9/1991, indiquaient entre autres: «lecture critique [...] d'un ou de plusieurs manuels pédagogiques, et d'un corpus d'articles [...] rédaction de la note de recherche ». Le programme de la session finale comportait : « rédaction de réponses, en français et en anglais, à une série de questions portant sur le sujet des cours, présentation et discussion de l'ensemble des travaux individuels, soutenance de la note de recherche ».

6. La proportion des étudiants francophones et anglophones, inscrits au DEA, était respectivement de $63 \%$ et $37 \%$.

7. Débat avec Jean-Claude Milner au Collège de France, 8 février 1993, «Où va la linguistique générale?».

\section{RÉSUMÉS}

En 1991-92, une première promotion a participé au Diplôme d'Étude Approfondie (DEA) de «Langue anglaise des spécialités scientifiques et techniques» (Université Bordeaux 2). L'auteur a demandé à tous les participants de répondre à un questionnaire autour du thème des "Contraintes et des libertés", le thème du colloque du GERAS en 1993. Les réponses aux questions, qui portaient sur l'espace, le temps, les aspects financiers et psychologiques, ont été regroupées à l'aide d'un tableur. Les réponses sont présentées sous forme de graphiques et de tableaux; elles sont brièvement commentées. Le questionnaire comportant des questions 
ouvertes, une analyse qualitative est proposée. L'objectif principal est de dresser un bilan de l'année d'étude vue par les utilisateurs.

For the first time in 1991-92, a group of students participated in a predoctoral programme in English for Specific Purposes, the Diplôme d'Étude Approfondie (DEA) «Langue anglaise des spécialités scientifiques et techniques» (University of Bordeaux 2). The author asked all the participants to fill in a questionnaire on the subject of "Constraint and Freedom", the theme of the GERAS 1993 Conference. The answers to the questions, which dealt with space, time, financing and psychological aspects, were collected with the help of a spreadsheet. The answers are presented with graphs and charts, and briefly commented upon. Since the questionnaire included openended questions, a qualitative analysis is presented. The main objective is to take stock of the year's study as seen from the users' point of view.

INDEX

Keywords : assessment, ESP, predoctoral programme

Mots-clés : bilan, DEA de langue anglaise, questionnaire

\section{AUTEUR}

\section{MONIQUE MÉMET}

IUT, Université Paris 12.m.memet@orange.fr 